\title{
CRESCIMENTO DE BAGAS DE CULTIVARES DE UVAS APIRÊNICAS TRATADAS COM CPPU E GA 3
}

\author{
VALTEMIR GONÇALVES RIBEIRO ${ }^{1}$ \\ JOÃO ALEXIO SCARPARE FILHO ${ }^{2}$
}

\begin{abstract}
RESUMO - As cultivares de uvas apirênicas (Vitis vinifera L.), via de regra, possuem bagas de tamanhos reduzidos, necessitando de ajustes no manejo para a melhoria da qualidade dos cachos, sendo a aplicação de reguladores de crescimento um dos tratamentos mais eficazes. Objetivou-se com o presente trabalho avaliar os efeitos do forchlorfenuron (CPPU: 0 e $10 \mathrm{mgL}^{-1}$ ) combinado ao ácido giberélico $\left(\mathrm{GA}_{3}: 0,25,50,75\right.$ e 100 $\mathrm{mgL}^{-1}$ ) durante o primeiro ciclo de produção das cultivares
\end{abstract}

Centennial Seedless, Flame Seedless e Thompson Seedless. As características avaliadas foram o comprimento, diâmetro e peso de bagas. Para a 'Centennial Seedless', maiores comprimentos, diâmetro e peso de bagas foram obtidos com $100 \mathrm{mgL}^{-1}$ de $\mathrm{GA}_{3}$; e a concentração de 100 $\mathrm{mgL}^{-1}$ de $\mathrm{GA}_{3}$ adicionada a $10 \mathrm{mgL}^{-1}$ de CPPU foi a mais responsiva para a 'Flame Seedless' e 'Thompson Seedless', observando-se atrasos na maturação, em termos de sólidos solúveis totais, para todas as cultivares.

TERMOS PARA INDEXAÇÃO: Uva para mesa, 'Centennial Seedless', 'Flame Seedless', 'Thompson Seedless', fisiologia da produção, Vitis vinifera.

\section{INCREASE OF THE CULTIVARS BERRY APIRENIC GROWTH OF SEEDLESS GRAPES TREATED WITH CPPU AND GA}

\begin{abstract}
The Seedless grapes (Vitis vinifera L.), have always decrease in berry size, requiring managements to increase cluster quality. The spray with growth regulators is one of most effective the treatment to enlarge berry size. The objective of this work was to determine the effects of Forchlorfenuron (CPPU: 0 e $10 \mathrm{mgL}^{-1}$ ) associated with Giberelic acid $\left(\mathrm{GA}_{3}: 0,25,50,75\right.$ e $\left.100 \mathrm{mgL}^{-1}\right)$ during the first cycle of production of the cultivars Centennial Seedless,
\end{abstract}

Flame Seedless and Thompson Seedless. The appraised characteristics were length, diameter and weight of berries. For Centennial Seedless, larger lengths, diameter and weight of berries were obtained with 100 $\mathrm{mL}^{-1}$ of $\mathrm{GA}_{3}$; and the concentration of $100 \mathrm{~mL}^{-1}$ of $\mathrm{GA}_{3}$ added to $10 \mathrm{~mL}^{-1}$ of CPPU was the most responsive for Flame and Thompson Seedless, being observed delays in maturation, in terms of total soluble solids, for all cultivars.

INDEX TERMS: Table grapes, forchlorfenuron, gibberellic acid, cytokine, crop physiology, Vitis vinifera.

\section{INTRODUÇÃO}

A produção de uvas apirênicas para mesa no Brasil carece de cultivares bem adaptadas e produtivas. A criação de cultivares com qualidades de cachos exigidas pelos mercados demanda tempo, tornando-se a introdução de cultivares a via mais rápida para a solução desse problema. No entanto, a maioria das cultivares já introduzidas têm apresentado baixa produção e qualidade de cachos, necessitando de ajustes no manejo (CAMARGO, 1998).
A `Centennial Seedless`é uma cultivar bem difundida na região noroeste paulista, produzindo em média um cacho por ramo, caracteristicamente grande, cheio, porém não compacto, e as bagas são alongadas e afiladas na ponta, esverdeadas, sabor neutro, crocantes e levemente aromáticas; porém, tem problemas de degrana e quando amadurecem, sob intenso calor, as bagas apresentam manchas na película. A ‘Flame Seedless`, obtida pelo Departamento de Agricultura dos Estados Unidos, em Fresno, Califórnia, é tida como promissora para a região do Vale do Submédio São Francisco

1. Engenheiro Agrônomo, Doutorando em Fitotecnia pela Escola Superior de Agricultura "Luiz de Queiroz" - Universidade de São Paulo - Departamento de Produção Vegetal (DPV/ESALQ-USP). Universidade do Estado da Bahia (UNEB). Av. Edgard Chastinet, s/n. Juazeiro, BA. 48900-000. valtemirribeiro@bol.com.br 2. Professor, Doutor do Departamento de Produção Vegetal da ESALQ/USP. jascarpa@carpa.ciagri.usp.br 
(BA/PE), chegando a produzir em torno de $18 \mathrm{t} / \mathrm{ha}$ (CAMARGO et al., 1997); seus cachos são médios, cônicos, moderadamente soltos, e as bagas são pequenas, arredondadas, avermelhadas, com textura firme, sabor neutro e doce. A 'Thompsons Seedless`, comparada às duas últimas, é a cultivar menos produtiva e adaptada aos nossos sistemas tropicais de cultivo; tem cachos grandes, alados, cilíndrico-alongados e compactos, e as bagas são esverdeadas ou levemente douradas quando maduras, textura firme, sabor neutro e doce (CAMARGO, 1998; POMMER, 1998; TERRA et al., 1998).

As bagas de uvas apirênicas tendem a ter tamanhos reduzidos pela ausência das sementes e de fitormônios que as façam crescer, sendo a aplicação de fitorreguladores de crescimento indispensável como parte do manejo para a melhoria da qualidade dos cachos.

$\mathrm{O}$ ácido giberélico $\left(\mathrm{GA}_{3}\right)$ tem sido muito utilizado para aumentar o tamanho das bagas. Atua na produção da -amilase, aumentando os teores de açúcares e conseqüentemente a pressão osmótica do suco celular, acarretando um maior influxo de água para o interior da célula, propiciando a sua elongação. O forchlorfenuron (CPPU) tem ação citocinínica, favorece a divisão e a expansão celular e atrasa o amadurecimento das bagas, tornando-as maiores (REYNOLDS et al., 1992; PIRES, 1998).

Sarig et al. (1998) avaliaram os efeitos do CPPU e do $\mathrm{GA}_{3}$ sobre as bagas das cultivares Thompson Seedless e Zeiny, e concluíram que os efeitos anatômicos causados pelo CPPU foram devidos a um aumento na divisão celular, resultando células menores e uma alta densidade celular seguida do engrossamento da película das bagas; em contrapartida, o $\mathrm{GA}_{3}$ provoca um aumento significativo no tamanho das células, especificamente daquelas localizadas na hipoderme. O thidiazuron (TDZ) é uma outra fenilurea que também tem sido utilizada com sucesso para o crescimento das bagas (REYNOLDS et al., 1992; CZERMAINSKI e CAMARGO, 1998), o que ressalta os benefícios não só das giberelinas como também das citocininas (WEAVER et al., 1966) sobre o crescimento de bagas da videira.

$\mathrm{O}$ uso do $\mathrm{GA}_{3}$ de forma isolada ou combinado com o CPPU tem favorecido a melhoria da qualidade das bagas em várias cultivares. Para a cultivar Itália, recomenda-se, para as condições do noroeste paulista, aplicar de 20 a $40 \mathrm{mgL}^{-1}$ aproximadamente 30 dias após o pleno florescimento (TERRA et al., 1998). Muñoz (1987) e Retamales et al. (1995) obtiveram no
Chile aumentos no tamanho das bagas da 'Thompson Seedless` com a aplicação de $40 \mathrm{mgL}^{-1}$ de $\mathrm{GA}_{3}$, e, de acordo com Pires (1998), a aplicação de $10 \mathrm{mgL}^{-1}$ tem sido a recomendada para as cultivares Redglobe e Thompson Seedless. Contudo, é um produto não registrado no Ministério da Agricultura, Pecuária e Abastecimento para utilização em videira (MIELE et al., 1998; LEÃO et al., 1999). Em trabalhos conduzidos com 'Perlette' e 'Flame Seedless' (DIAZ e MALDONADO, 1992), 'Sovereign Coronation' e 'Sommerland' (REYNOLDS et al., 1992) e 'Perlette' (LEÃO et al., 1999), foram observados efeitos maximizadores pelo uso conjunto do CPPU com o $\mathrm{GA}_{3}$.

Objetivou-se com o presente trabalho estudar o efeito do CPPU e do $\mathrm{GA}_{3}$ sobre o crescimento de bagas das cultivares $V$. vinifera L. Flame Seedless, Centennial Seedless e Thompson Seedless, durante o seu primeiro ciclo de produção, em Porto Feliz, SP.

\section{MATERIAL E MÉTODOS}

O experimento foi executado em vinhedo comercial localizado no município de Porto Feliz, SP, situado a $2312^{\prime} \mathrm{S}, 47$ 31'W e altitude de $540 \mathrm{~m}$. As cultivares Centennial Seedless, Flame Seedless e Thompson Seedless, durante o primeiro ciclo de produção, foram conduzidas em sistema de latada, com dois braços primários, sentidos linha, tendo sido enxertadas no portaenxerto IAC-572 'Jales', no espaçamento 3,0x3,60 m.

Após as podas, realizadas em agosto de 2000, aplicou-se nas gemas, na forma de pincelamento, cianamida hidrogenada a 2,5\% para quebra de dormência e uniformidade da brotação. Com 15 dias após o pleno florescimento, os cachos foram pulverizados com CPPU: 0 e $10 \mathrm{mgL}^{-1}$, em associação com o ácido giberélico nas doses de $0,25,50,75$ e $100 \mathrm{mgL}^{-1}$, em todas as combinações possíveis. $\mathrm{O}$ experimento foi conduzido em delineamento de blocos casualizados, em esquema fatorial $2 \times 5$, com quatro repetições, com uma planta por parcela.

As avaliações foram realizadas pelo comprimento, diâmetro e peso médio de 100 bagas, retiradas de um total de cinco cachos amostrados por planta. Para a análise do teor de sólidos solúveis totais ( ${ }^{\circ}$ Brix), foram coletadas de cada cacho uma baga da região apical, duas da central e uma da basal, compondo-se, portanto, amostras de 20 bagas para cada tratamento. As medições de todos os tratamentos foram realizadas em um mesmo momento, a partir da constatação do mínimo recomendável de $14^{\circ}$ Brix para a colheita (ALMEIDA, 
2001), verificado no tratamento-controle. Essa medida foi tomada em razão da contingência de insetos e pássaros na área experimental, por ter-se conduzido o experimento sem o uso de tela protetora.

Para fins de análise estatística, as variáveis comprimento, diâmetro e peso médio das bagas das três cultivares foram transformadas $\mathrm{x}+0,5$ e interpretadas por meio de análise de variância e regressão, baseandose para a escolha dos modelos a significância dos coeficientes de regressão, utilizando-se o teste " $\mathrm{t}$ " $(<0,05)$ e o coeficiente de determinação $\left(R^{2}>0,70\right)$.

\section{RESULTADOS E DISCUSSÃO}

O CPPU não teve efeito significativo sobre o diâmetro e o peso de baga da 'Centennial Seedless`, e a combinação com $\mathrm{GA}_{3}$ também não foi significativa para o peso de baga da 'Thompson Seedless`; contudo, em termos gerais, os efeitos dos dois fatores foram altamente significativos $(<0,01)$, como pode ser observado nas figuras correspondentes a cada característica avaliada.
A cultivar Centennial Seedless possui naturalmente bagas superiores às da 'Flame Seedless' e 'Thompson Seedless', em comprimento, diâmetro e peso, que podem ser verificados nos tratamentos com a ausência dos produtos (Figuras 1, 2 e 3). A concentração de $10 \mathrm{mgL}^{-1}$ de CPPU interagiu positivamente com os níveis utilizados de $\mathrm{GA}_{3}$ e promoveu ganhos maiores do que com o $\mathrm{GA}_{3}$ aplicado isoladamente, para a característica comprimento de baga da cultivar 'Centennial Seedless` (Figura 1), para o diâmetro de baga em 'Flame Seedless`e 'Thompson Seedless (Figura 2), e para o peso de baga em `Flame Seedless`e `Centennial Seedless` (Figura 3). Resultados semelhantes foram observados por Lee et al. (1996) no aumento da massa dos frutos, cachos e diâmetro das bagas em 'Kyoho', por imersão em mistura de $\mathrm{GA}_{3}$ a $25 \mathrm{mgL}^{-1}$ e citocinina a $10 \mathrm{mgL}^{-1}$. Nickell (1985), Diaz e Maldonado (1992), Reynolds et al. (1992), Sarig et al. (1998) e Leão et al. (1999) atribuíram o melhor desempenho para a mistura como conseqüência do sinergismo do $\mathrm{GA}_{3}$ com a ação citocinínica do CPPU sobre o crescimento das bagas.

$$
\begin{aligned}
& Y^{`}{ }^{` C e n t e n n i a l ~ s d s ` ~ C P P U ~} 0=2,827357-0,0005556 \mathrm{x}+0,00004649 \mathrm{x}^{2} \\
& \mathrm{R}^{2}=0,97 \\
& Y^{` C e n t e n n i a l ~ S e e d l e s s ` ~ C P P U ~} 10 \mathrm{mgL}^{-1}=2,889057+0,0101186 \mathrm{x}-0,00014589 \mathrm{x}^{2} \\
& \mathrm{R}^{2}=0,74 \\
& \text { Y`Flame Seedless`CPPU } 0=1,341689+0,0059899 \mathrm{x}-0,00005683 \mathrm{x}^{2} \\
& \mathrm{R}^{2}=0,95 \\
& Y^{`} \text { Flame Seedless` CPPU } 10 \mathrm{mgL}^{-1} \quad(\text { média geral })=1,62 \mathrm{~cm} \\
& Y^{` T h o m p s o n ~ S e e d l e s s ` C P P U ~} 0=1,590429+0,0052137 \mathrm{x}-0,00004526 \mathrm{x}^{2} \\
& \mathrm{R}^{2}=0,87 \\
& Y^{`} \text { Thompson Seedless` CPPU } 10 \mathrm{mgL}^{-1} \text { (média geral) }=1,94 \mathrm{~cm}
\end{aligned}
$$

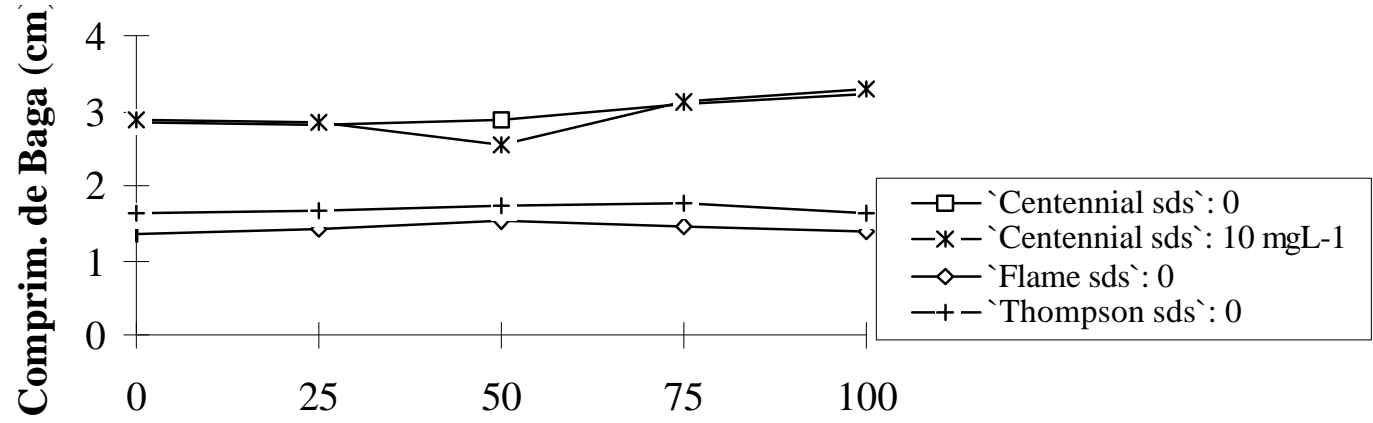

\section{Ácido Giberélico (mgL-1)}

FIGURA 1 - Efeito do CPPU e GA 3 sobre o comprimento de bagas das cultivares Centennial Seedless, Flame Seedless e Thompson Seedless. 
Quando aplicado isoladamente em 'Centennial Seedless`, o $\mathrm{GA}_{3}$ mostrou resultados semelhantes à mistura com CPPU, na concentração de $100 \mathrm{mgL}^{-1}$. Isso pode ser observado nas Figuras 1, 2 e 3, para as características comprimento, diâmetro e peso de baga, atingindo respectivamente médias de $3,2 \mathrm{~cm}, 2,0 \mathrm{~cm}$ e 8,4 g. Evidenciou-se, dessa forma, que mesmo tendo a concentração de $10 \mathrm{mgL}^{-1}$ de CPPU interagido positivamente com o $\mathrm{GA}_{3}$, o CPPU tornou-se dispensável para aumentar o crescimento e o peso de bagas dessa cultivar.

Esses resultados são diferentes dos de Pires (1998), em que o melhor tratamento foi obtido a 25 $\mathrm{mgL}^{-1}$ de $\mathrm{GA}_{3}$; entretanto, Reynolds et al. (1992) utilizaram com sucesso a concentração de $100 \mathrm{mgL}^{-1}$ de $\mathrm{GA}_{3}$ para cachos da cultivar Sovereign Coronation. Assim, sob diferentes particularidades de cultivo, cultivares e climas devem ser levados em consideração para uma melhor eficiência dos fitorreguladores.

Para a 'Flame Seedless', o comprimento máximo de baga $(1,5 \mathrm{~cm})$ foi conseguido com $52,7 \mathrm{mgL}^{-1}$ de $\mathrm{GA}_{3}$. Para diâmetro e peso da baga, a associação de 10
$\mathrm{mgL}^{-1}$ de CPPU com o $\mathrm{GA}_{3}$ sempre promoveu ganhos superiores aos efeitos isolados do $\mathrm{GA}_{3}$, sendo as concentrações de 25 e $100 \mathrm{mgL}^{-1}$ de $\mathrm{GA}_{3}$ aquelas que igualmente interagiram com o CPPU, maximizando o diâmetro e o peso da baga, respectivamente a $1,8 \mathrm{~cm}$ e $3,9 \mathrm{~g}$.

Manteve-se uma tendência linear de aumentos no diâmetro da baga da 'Thompson Seedless' com o CPPU a $10 \mathrm{mgL}^{-1}$ para com todos os níveis de $\mathrm{GA}_{3}$, verificando-se $1,8 \mathrm{~cm}$ com a concentração de $100 \mathrm{mgL}^{-1}$ de $\mathrm{GA}_{3}$. Já para a característica comprimento de baga, somente para a ausência do CPPU verificaram-se ajustes de dados a um modelo $\left(\mathrm{R}^{2}>0,70\right)$, computando-se o comprimento $1,7 \mathrm{~cm}$ com $57,6 \mathrm{mgL}^{-1}$ de $\mathrm{GA}_{3}$. Para o peso de baga, não foram observadas diferenças significativas com a aplicação de CPPU e $\mathrm{GA}_{3}$, o que também foi verificado por Joublan et al. (1995) com a cultivar Moscatel Rosada usando-se $\mathrm{GA}_{3}$ a $35 \mathrm{mgL}^{-1}$ e CPPU a 0, 2, 4 e $6 \mathrm{mgL}^{-1}$; porém, Rematales et al. (1995) obtiveram aumentos significativos no peso de baga da 'Thompsons Seedless' com a combinação desses dois fitorreguladores.

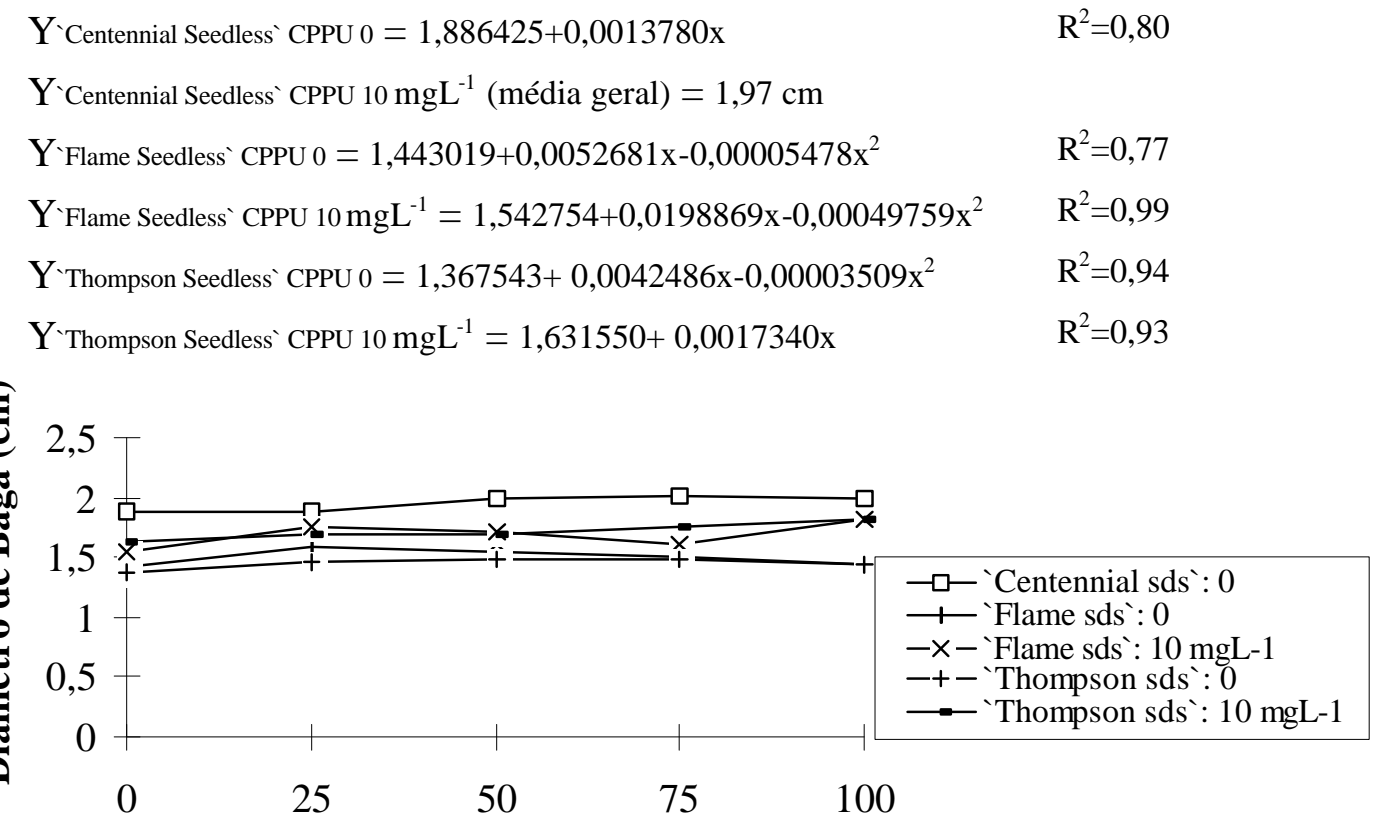

\section{Ácido Giberélico (mgL-1)}

FIGURA 2 - Efeito do CPPU e GA 3 sobre o diâmetro de bagas das cultivares Centennial Seedless, Flame Seedless e Thompson Seedless. 

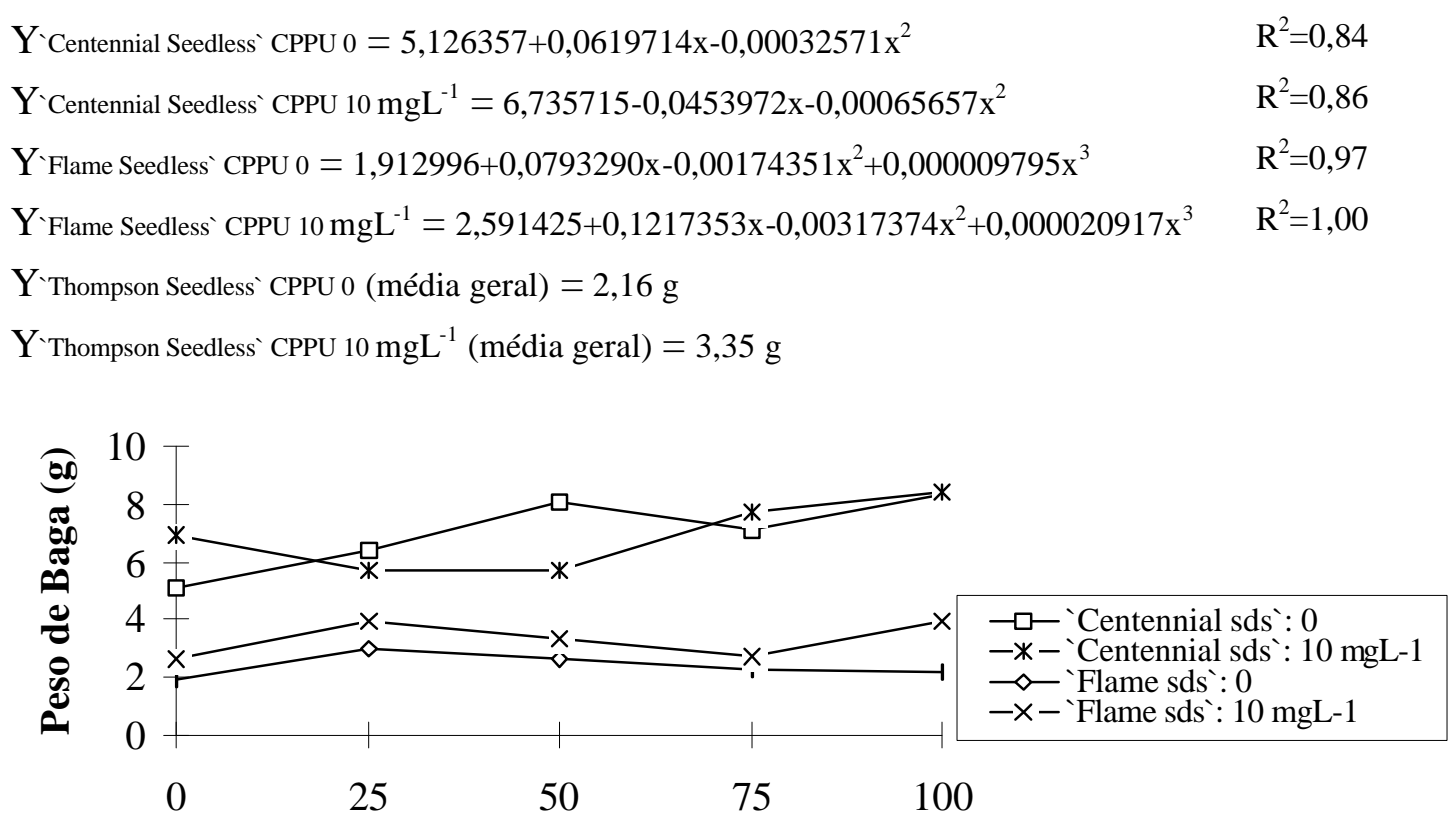

Ácido Giberélico (mgL-1)

FIGURA 3 - Efeito do CPPU e $\mathrm{GA}_{3}$ sobre o peso de bagas das cultivares Centennial Seedless, Flame Seedless e Thompson Seedless.

Portanto, conclui-se que os tratamentos com fitorreguladores foram efetivos para a melhoria da qualidade das bagas das três cultivares. De acordo com as normas de classificação de uvas finas de mesa (ALMEIDA, 2001), em média, as bagas da `Centennial Seedless` situaram-se no calibre 21 para diâmetro de bagas com $100 \mathrm{mgL}^{-1}$ de $\mathrm{GA}_{3}$, enquanto as da 'Flame Seedless`e 'Thompson Seedless` atingiram calibre 19, pelos efeitos do tratamento com $10 \mathrm{mgL}^{-1}$ de CPPU e $100 \mathrm{mgL}^{-1}$ de $\mathrm{GA}_{3}$.

Na Figura 4 verifica-se uma tendência generalizada de as bagas da cultivar Flame Seedless possuírem ${ }^{\circ}$ Brix naturalmente superiores às da 'Centennial Seedless` e ‘Thompson Seedless', com ou sem a aplicação dos fitorreguladores. Como citado anteriormente, pelas contingências de pássaros e insetos na área experimental, fazendo com que o experimento fosse colhido ao se atingir $14{ }^{\circ}$ Brix, infere-se que os teores de sólidos solúveis totais poderiam ser superiores aos observados, devido o retardamento no amadurecimento das bagas provocado pelos efeitos dos tratamentos.

Os tratamentos 2 e 5, com apenas ácido giberélico, proporcionaram respectivamente para as cultivares
Flame Seedless, Centennial Seedless e Thompson Seedless médias de 13,7, 12,8 e 12,4 Brix, e, com associações com $10 \mathrm{mgL}^{-1}$ de CPPU nos tratamentos 6 a 10 , reduziram-se ainda mais os teores de ${ }^{\circ}$ Brix para 12,1, 10,6 e 8,6; acentuando-se, portanto, as quedas no ${ }^{\circ}$ Brix com a adição do CPPU. Esse fato foi mais bem visualizado pelo tratamento 7, com CPPU $10 \mathrm{mgL}^{-1}+$ $\mathrm{GA}_{3} 25 \mathrm{mgL}^{-1}$, quando os teores de ${ }^{\circ}$ Brix das três cultivares foram reduzidos em média para aproximadamente 9,1 .

Atraso na maturação, em termos da redução dos teores de sólidos solúveis totais, tem sido constatado em diversos trabalhos (DIAZ e MALDONADO, 1992; REYNOLDS et al., 1992; REMATALES et al., 1995) pela aplicação conjunta do forchlorfenuron com o ácido giberélico.

Resultados semelhantes foram encontrados por Joublan et al. (1995), e Leão et al. (1999) encontraram diferenças de até oito dias no amadurecimento de bagas no primeiro ciclo de produção da cultivar Perlette, em Petrolina, $\mathrm{PE}$, devido à combinação de $\operatorname{CPPU}\left(0,5,10\right.$ e $\left.20 \mathrm{mgL}^{-1}\right)$ com $\mathrm{GA}_{3}(0,10$ e 40 $\left.\mathrm{mgL}^{-1}\right)$. 


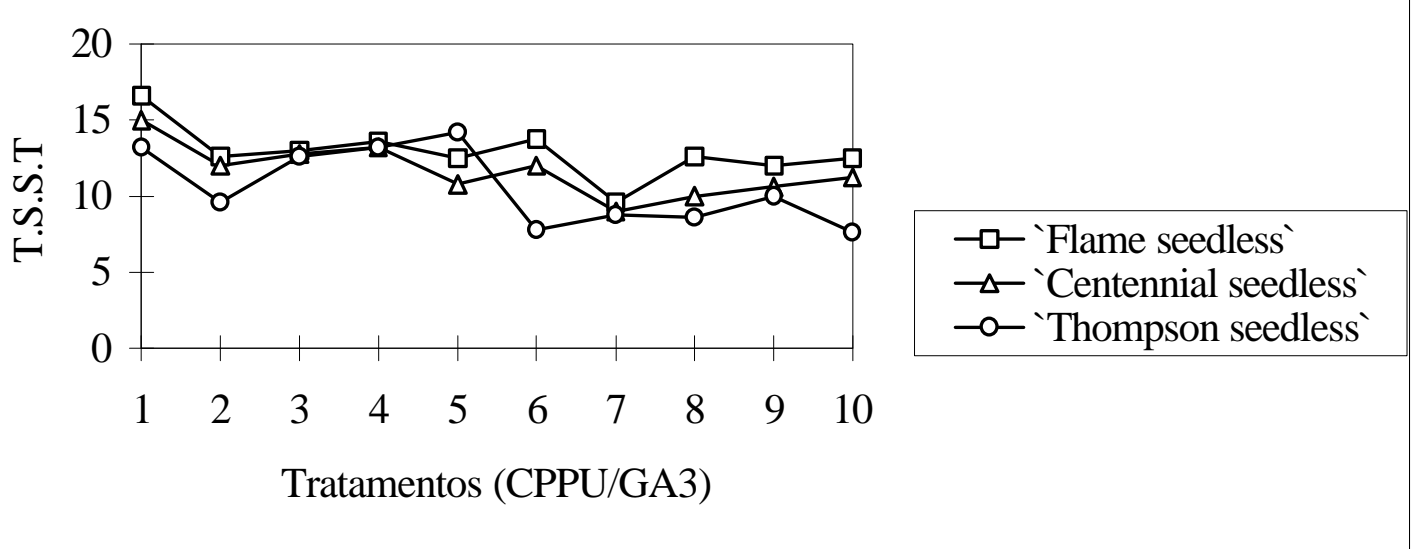

FIGURA 4 - Efeito do CPPU e GA 3 (T1:0-0; T2:0-25; T3:0-50; T4:0-75; T5:0-100; T6:10-0; T7:10-25; T8:10-50; T9:10-75 e T10:10-100 $\mathrm{mgL}^{-1}$, respectivamente) sobre os teores de sólidos solúveis totais no mosto das cultivares Centennial Seedless, Flame Seedless e Thompson Seedless.

\section{CONCLUSÕES}

As características comprimento, diâmetro e peso de bagas das cultivares Centennial Seedless, Flame Seedless e Thompsons Seedless são melhoradas com aplicações de CPPU e $\mathrm{GA}_{3}$; contudo, para uma recomendação comercial das concentrações de fitorreguladores, em face das variações climáticas ao longo dos anos, seriam necessárias avaliações em mais ciclos produtivos.

\section{AGRADECIMENTO}

Ao técnico agrícola Sr. David, do Departamento de Horticultura da USP/ESALQ, pelos auxílios operacionais dispensados durante a execução dos experimentos.

\section{REFERÊNCIAS BIBLIOGRÁFICAS}

ALMEIDA, G. V. B. Embalagem e comercialização de uva. In: BOLIANI, A. C.; CORRÊA, L. de S. (Eds.). Cultura de uvas de mesa: do plantio à comercialização. Ilha Solteira: UNESP, 2001. cap. 15, p. 281-316.

CAMARGO, U. A. Cultivares para a viticultura tropical no Brasil. Informe Agropecuário, Belo Horizonte, v. 19, p. 15-19, 1998.

CAMARGO, U. A.; MASHIMA, C. H.; CZERMAINSKI, A. B. C. Avaliação de cultivares de uvas apirênicas no Vale do São Francisco. Bento Gonçalves: EMBRAPA Uva e Vinho, 1997. 8 p. (Comunicado Técnico, 26).
CZERMAINSKI, A. B. C.; CAMARGO, U. A. Influência do ácido giberélico e do thidiazuron sobre a qualidade da uva 'Vênus'. In: CONGRESSO BRASILEIRO DE FRUTICULTURA, 15., 1998, Poços de Caldas. Anais... Poços de Caldas: Sociedade Brasileira de Fruticultura, 1998. p. 747.

DIAZ, H. D.; MALDONADO, L. A. Forchlorfenuron effects on berry size and maturity of 'Perlette' and 'Flame Seedless' grapes. Proceedings Plant Growth Regional Society American, [S.1.], v. 19, p. 123-128, 1992.

JOUBLAN, M. J. P.; MERINO, H. R.; WILCHENS, E. R.; MEDINA, E. Efecto del CPPU y acido giberelico en frutos de vid cv. Moscatel Rosada. Agro Ciência, Concepcion, v. 11, p. 119-127, 1995.

LEÃO, P. C. de S.; LINO JÚNIOR, E. da. C.; SANTOS, E. da S. Efeitos do CPPU e ácido giberélico sobre o tamanho de bagas da uva 'Perlette' cultivada no vale do São Francisco. Revista Brasileira de Fruticultura, Cruz das Almas, v. 21, p. 74-78, 1999.

LEE, C. H.; HAN, D. H.; KIM, S. B. Effects of GA and Fulmet (KT-30) on fruit set and quality in 'Kyoho' grape. Journal of the Korean Society for Horticultural Science, [S.1.], v. 37, p. 686-690, 1996.

MIELE, A.; DALL'AGNOL, I.; BIANCHI, A. Efeito do CPPU no tamanho da baga e na composição da uva 'Itália'. In: CONGRESSO BRASILEIRO DE FRUTICULTURA, 15., 1998, Poços de Caldas. 
Anais... Poços de Caldas: Sociedade Brasileira de Fruticultura, 1998. p. 742.

MUÑOZ, I. El cultivo de la uva de mesa: algunos aspectos de manejo como fatores de calidad. Santiago: Instituto de Investigaciones Agropecuarias-Estacion Experimental La Platina, 1987. 36 p.

NICKELL, L. G. New plant growth regulator increases grape size. Proceedings Plant Growth Regional Society American, [S.1.], v. 12, p. 1-7, 1985.

PIRES, E. J. P. Emprego de reguladores de crescimento em viticultura tropical. Informe Agropecuário, Belo Horizonte, v. 19, p. 40-43, 1998.

POMMER, C. V. Uvas sem sementes. O Agronômico, Campinas, v. 47, p. 38-39, 1998.

REMATALES, J.; BANGERTH, F.; COOPER, T.; CALLEJAS, R. Effects of CPPU and $\mathrm{GA}_{3}$ on fruit quality of Sultanina table grape. Acta Horticulturae, The Hague, n. 394, p. 149-157, 1995.
REYNOLDS, A. G.; WARDLE, D. A.; ZUROWSKI, C.; LOONEY, N. E. Phenylureas CPPU and thidiazuron affect yield compnents, fruit composition, and storage potential of four Seedless grape selections. Journal of the American Society for Horticultural Science, [S.1.], v. 117, p. 85-89, 1992.

SARIG, P.; ZUTKHI, Y.; LISKER, N.; SHKELERMAN, Y.; BEN-ARIE, R. Natural and induced resistance of table grapes to bunch rots. Acta Horticulturae, The Hague, n. 464, p. 65-70, 1998.

TERRA, M. M.; PIRES, E. J. P.; NOGUEIRA, N. A. M. (Coords.). Tecnologia para produção de uva Itália na região noroeste do Estado de São Paulo. Campinas: CATI, 1998. 81 p. (Documento Técnico, 97).

WEAVER, R. J.; OVERBEEK, J. van; POOL, R. M. Effect of kinins on fruit set and development in Vitis vinifera. Hilgardia: A Journal of Agricultural Science, Berkeley, v. 35, p. 181-201, 1966. 